http://revistainvestigacionacademicasinfrontera.com

Recibido el 20 de septiembre de 2018 Dictaminado mediante arbitraje favorablemente 18 de diciembre de 2018

\title{
Evaluación de la aptitud física en adultos mayores miembros del programa INAPAM de la ciudad de Navojoa Sonora.
}

\author{
*Lic. José Samuel Borbón Vega \\ **Mtro Jesús Santos Cantúa Quintero \\ ***Lic. Emmanuel Josef García Medina \\ ****Lic. Marco Alejandro Molina Rodríguez
}

\section{INSTITUCIONES:}

Universidad Estatal de Sonora

Instituto Tecnológico de Sonora

\section{Resumen}

La investigación se centró en la evaluación de las aptitudes físicas en miembros del programa INAPAM, para mejorar aptitudes física y calidad de vida en los adultos mayores. Se implementó la metodología, (Rikli \& Jones, 1999) la cual incluye una batería de condición funcional para personas mayores, conformada por los siguientes test, de equilibrio estático, de flexibilidad de las extremidades superiores y de agilidad. Los resultados arrojaron para el test de equilibrio estático con la pierna izquierda una media de 3.554. Con respecto al mismo test pero con la pierna derecha en la primera evaluación la media fue de 4.577, Para el test de flexibilidad de extremidades superiores en la primera evaluación la media fue de 2.454. Por ultimo en el test de agilidad en la primera evaluación la media fue de 1.315. Se concluye que los adultos mayores de INAPAM tienen una aptitud física deficiente.

Palabras clave: Aptitud física, Adultos Mayores, Ejercicio Físico, Envejecimiento, Vejez.

* Lic. José Samuel Borbón Vega: Entrenador INAPAM Navojoa.

**Mtro Jesús Santos Cantúa Quintero: Profesor de tiempo completo UES Navojoa.

*** Lic. Emmanuel Josef García Medina: Profesor de tiempo completo ITSON Navojoa.

**** Lic. Marco Alejandro Molina Rodríguez: Coordinador de deportes ITSON Navojoa. 


\section{http://revistainvestigacionacademicasinfrontera.com}

\section{Introducción}

El proceso de envejecer se asocia a una fase vital en la que progresivamente el individuo pasa de la plenitud física, social y mental a la más absoluta decrepitud (Sarabia, 2009), dicho de otra manera el envejecimiento es un proceso que se vive desde el nacimiento y se caracteriza por diferentes cambios en niveles físicos, mentales, individuales y colectivos. Estos cambios definen a las personas cuando ya están mayores, pero se debe ver como un proceso natural, inevitable y no necesariamente ligado a estereotipos; como una etapa de la vida apta para crecer y continuar aprendiendo" (Daza, 2011).

Otra definición de envejecimiento propuesta por el Instituto para la Atención de los Adultos Mayores de la Ciudad de México se indica que es un proceso de cambios a través del tiempo, natural, gradual, continuo, irreversible y completo. Estos cambios se dan a nivel biológico, psicológico y social, y están determinados por la historia, la cultura y la situación económica, de los grupos y las personas (IAAM, 2018).

Analizando los distintos conceptos de envejecimiento se extraen de las teorías encontramos que el concepto del envejecimiento normal se orienta por la norma típica, como puede ser el envejecimiento sin enfermedades crónicas, que sería lo contrario del envejecimiento patológico (Havighurst, 1948).

Con respecto a la vejez se puede definir como el cambio fisiológico que sufre el individuo, cuyo término inevitablemente es la muerte (Ramos, Meza, Maldonado, Ortega, \& Hernandez, 2009).

Por lo tanto la vejez es una etapa de la vida, la última. Está relacionada con la edad, es decir, el tiempo que transcurre desde el nacimiento hasta el momento actual, y cómo se ha vivido durante ese tiempo (IAAM, 2018). 


\section{http://revistainvestigacionacademicasinfrontera.com}

A sí mismo la Organización Mundial de la Salud (OMS, 2015) y la Organización Panamericana de la Salud (OPS, 2015) definen a un adulto mayor funcionalmente sano como el anciano capaz de enfrentar este proceso de cambio con un nivel adecuado de adaptación funcional y de satisfacción personal.

Hay que mencionar que el adulto mayor es un ser humano que integra un grupo de riesgos para múltiples enfermedades y disfunciones orgánicas, derivadas del proceso natural de envejecimiento. Este hecho, hace del adulto mayor una persona frágil, cuya integridad física, psicológica, social y espiritual, se ve en constante riesgo de ser afectada por factores externos (Ceballos, Alvarez, \& Medina, 2012; Quevedo, 2011).

Se considera como adultos mayores a quienes tienen una edad mayor o igual de 60 años en los países de vías de desarrollo y de 65 años o más a quienes viven en países desarrollados. Estas diferencias cronológicas obedecen a las características socio-económicas que tienen unos y otros países (Torres, 2003).

Las personas de 60 a 74 años son considerados de edad avanzada, de 75 a 90 años viejas o ancianas, y los que sobre pasan los 90 años se les denomina grandes, viejos o longevos. A todo individuo mayor de 60 años se le llamara de forma indistinta persona de la tercera edad (Guzman, 2010).

Así pues los adultos mayores son personas que por su condición física y los cambios físicos y biológicos que se presentan en esta etapa de la vida pierden la funcionalidad y la autonomía esto hace que se les dificulte la realización de ejercicio, provocando así cambios en sus cuerpos e incrementando los periodos de depresión y agotamiento, aunque algunos signos son a causa de la edad, la mayoría de estos se presentan por el tiempo de ocio que desarrollan y la inactividad física (IAAM, 2018; (Moreno, 2005). 


\section{http://revistainvestigacionacademicasinfrontera.com}

Otra variable de importancia para la investigación es la actividad física en cuanto a esta variable en personas de edad avanzada o de la tercera edad se tiene la idea que cuando una persona llega a la jubilación lo primero que hará ahora será descansar, esto incluye la inactividad física y, poco a poco, la persona va moviéndose menos y sin darse cuenta pierde funcionabilidad, porque el ejercicio es consustancial a la naturaleza humana y estamos diseñados para movernos" (Garcia, 2010).

La Comisión europea indica que la actividad física en personas de edad avanzada es cualquier movimiento corporal asociado con la contracción muscular que incrementa el gasto de energía por encima de los niveles de reposo", por ejemplo actividad física en el tiempo de ocio (incluyendo la mayor parte de las actividades deportivas y el baile), actividad física ocupacional, actividad física en o cerca del hogar y actividad física conectada con el transporte (UE, 2008).

Sin embargo para la, (OMS, 2018) la actividad física se considera como movimiento corporal producido por los músculos esqueléticos que exija gasto de energía, destacando que en los adultos mayores la "actividad física" no debe confundirse con el "ejercicio". Ya que es una variedad de actividad física planificada, estructurada, repetitiva y realizada con un objetivo relacionado con la mejora o el mantenimiento de uno o más componentes de la aptitud física.

Para los adultos de este grupo de edades, la actividad física consiste en actividades recreativas o de ocio, desplazamientos (por ejemplo, paseos caminando o en bicicleta), actividades ocupacionales (cuando la persona todavía desempeña actividad laboral), tareas domésticas, juegos, deportes o ejercicios programados en el contexto de las actividades diarias, familiares y comunitarias (OMS, 2018).

En vinculación con la actividad física encontramos al ejercicio físico que es toda actividad física planificada y estructurada que se realiza con la intención de mejorar o mantener una o varios aspectos de la condición física, entendiendo por condición física el desarrollo o adquisición de las 


\section{http://revistainvestigacionacademicasinfrontera.com}

capacidades físicas básicas, es decir, resistencia cardiovascular, flexibilidad, fuerza muscular, equilibrio y coordinación (Perez \& Perez, 2013).

La aptitud física es definida como un conjunto de atributos que las personas tienen o alcanzan y se asocia a la capacidad de realizar actividad física, su evaluación se realiza por medio de test físicos específicos y debe de llevarse un control periódico de la misma (Cossio \& Arruda, 2009; Laverde, Esguerra, Espinosa, \& Lozano, 2011).

La evaluación del nivel de aptitud física, permite también un mantenimiento de la funcionalidad corporal de forma adecuada, posibilitando la autonomía personal en la práctica del ejercicio físico, así como en tareas cotidianas que requieren de la misma lo que conduce al mejoramiento de la salud (Farinola, 2010; Laverde, Esguerra, Espinosa, \& Lozano, 2011).

Los medios más comunes para evaluar la aptitud física en la población en general son los test físicos que sirven para valorar la capacidad aeróbica, la fuerza y la velocidad (Guillen, Bueno, Gutierrez, \& Guerra, 2012). Sin embargo a través de la historia, el desarrollo de la cultura deportiva ha sido explorado continuamente, producto de la necesidad de desarrollar nuevas tendencias para el aumento de las capacidades físicas e incrementar el nivel de entrenamiento óptimo para vivir saludable.

\section{Método}

\section{Población y muestra.}

La población estuvo constituida por 18 sujetos, de los cuales 16 son del sexo femenino y 2 del sexo masculino, entre el rango de edad desde los 60 hasta los 94 años.

La muestra estuvo constituida por 13 sujetos de los cuales, 12 son del sexo femenino, y 1 del sexo masculino, entre el rango de edad desde los 60 hasta los 78 años. 


\section{http://revistainvestigacionacademicasinfrontera.com}

\section{Sujetos.}

Los sujetos a investigar son adultos mayores pertenecientes al programa de INAPAM, de las comunidades de Agiabampo y Pueblo Mayo.

\section{Diseño de investigación.}

La investigación es cuantitativa con un diseño descriptivo.

\section{Método.}

Batería de test físicos.

Los test que se aplicaron a los sujetos de investigación fueron tomados de la batería de condición funcional para personas mayores, propuesto por (Rikli \& Jones, 1999) a continuación se describen cada uno de ellos:

Test de equilibrio estático (Mantenerse en el sitio sobre un pie).

Protocolo: El/la participante deberá colocar sus manos en las caderas y permanecer apoyado sobre la planta de un pie. El otro pie permanecerá apoyado sobre el tobillo del pie sobre el que se sustenta.

El/la evaluador/a realiza una demostración y permite al/la participante una prueba de ensayo para que después realice 4 intentos (dos con cada pie). El pie de apoyo deberá ir alternándose. El test comenzará cuando el participante levante el pie del suelo y lo coloque sobre el tobillo del pie sobre el que se sustenta y en caso de no terminar antes el test finalizará después de 60 segundos. Se registra el tiempo a la décima de segundo más próxima.

En el caso de que el /la participante esté realizando la prueba de manera incorrecta, el/la evaluador/a puede corregir al/la participante durante la prueba de ensayo. Si las manos del/de la participante se separan de las caderas en el segundo o siguientes intentos, el/la evaluador/a deberá anotarlo y parar el cronómetro en ese momento. 


\section{http://revistainvestigacionacademicasinfrontera.com}

Posibles problemas en la ejecución:

Algunos de los problemas que pueden surgir al realizar esta prueba son:

Las manos se separan de las caderas

El pie se separa del tobillo y queda al aire

La punta de los dedos del pie no apoyado toca el suelo

Puntuación: Se considerará 60 segundos como la puntuación máxima para cada una de las piernas, marcando con un círculo el mejor de los 4 intentos. Se registrará el mejor tiempo de los intentos con la pierna derecha o la izquierda.

Test de flexibilidad de las extremidades superiores (Rascarse la espalda)

Protocolo: En una posición de pie, el/la participante coloca una mano preferida detrás del hombro del mismo lado, con la palma hacia abajo y los dedos extendidos, alcanzando la mitad de la espalda hacia abajo (con el codo dirigido hacia arriba). El/la participante coloca la otra mano detrás de la espalda, palma hacia fuera, alcanzando tan lejos como le sea posible en un intento para tocar o superponer los dedos medios (o los dedos más largos) extendidos de ambas manos.

Sin mover las manos del participante, el evaluador ayuda para ver que los dedos medios de cada mano se dirigen el uno hacia el otro. No se permite que el/la participante agarre o junte los dedos y tire. El evaluador NO puede ayudar al participante (estirando o empujando las manos) durante la ejecución del test.

Después de la demostración, por el/la evaluador/a, se deja al participante un tiempo de prueba y posteriormente realiza el test con dos intentos (uno con cada mano). 


\section{http://revistainvestigacionacademicasinfrontera.com}

Puntuación: Se mide la distancia de superposición o entre la punta de ambos dedos medios (o los más largos) al centímetro más próximo. Se concede una puntuación 8 negativa (-) para representar la distancia que falta para que se toquen los dedos; se concede una puntuación positiva (+) para representar la distancia de superposición. Registre ambos valores y marque con un círculo el mejor valor y utilícelo para evaluar la ejecución de la prueba. Asegúrese de registrar "menos" (-) o "más" (+) en la ficha de registro.

Observación: Para facilitar la medida conviene situar la regla por debajo de las manos (entre la espalda y las manos).

Test de agilidad (Test de levantarse, caminar (2`45 m y volver a sentarse)

Protocolo: La prueba comienza con el/la participante sentado/a completamente en la silla (posición erguida), las manos en los muslos y la planta de los pies apoyadas en el suelo (con un pie ligeramente adelantado). A la señal de YA!! El participante se levanta de la silla (se permite empujar los muslos), camina tan rápido como le sea posible alrededor del cono (por cualquiera de los lados), y regresa a la silla. Debería decirse al/la participante que es una prueba cronometrada y que debe realizarse tan rápido como sea posible (sin correr) alrededor del cono y regresar al cajón. El/la evaluador/a debe servir como referencia, estando de pie a mitad de la distancia entre la silla y el cono, listo para ayudar al participante en caso de que pierda el equilibrio. Para que el puntaje sea confiable, el evaluador debe poner en marcha el cronómetro a la señal de i¡YA!! ; Haya comenzado a moverse el/la participante o no, y parar el cronómetro en el momento exacto en el que el/la participante se siente en la silla.

Después de una demostración, el/la participante realiza dos veces el test. Se debe recordar a los participantes que el cronómetro no se para hasta el momento exacto en que los participantes se sientan en el cajón. 
Año 11.

Revista de Investigación

Núm. 28

Académica sin Frontera

ISSN: 2007-8870

\section{http://revistainvestigacionacademicasinfrontera.com}

Puntuación: La puntuación es el tiempo transcurrido desde la señal ¡¡YA!! ; Hasta que el/la participante regresa a la posición sentada en el cajón. Registre la puntuación de ambos test a la décima de segundo más próxima y rodee con un círculo la puntuación mejor (el menor tiempo). La mejor puntuación es utilizada para evaluar el rendimiento.

Observación: La distancia se tomará considerando el lado del cono más distante del borde delantero del cajón o de la silla.

\section{Análisis de Datos.}

Los datos se analizaron en el programa estadístico IBM SPSS Statistics 23. Se realizó un análisis descriptivo.

\section{Resultados}

\section{Resultados evaluación batería de condición funcional para personas mayores.}

A continuación se observan los resultados obtenidos de la primera evaluación del programa de entrenamiento para adultos mayores, usando la batería de condición funcional para personas mayores propuesto por (Rikli \& Jones, 1999). 
Núm. 28

ISSN: 2007-8870

\section{http://revistainvestigacionacademicasinfrontera.com}

Tabla 1. Evaluación estadísticos descriptivos.

\begin{tabular}{|c|c|c|c|c|c|}
\hline & $\mathrm{N}$ & Mínimo & Máximo & Media & $\begin{array}{l}\text { Desviación } \\
\text { estándar }\end{array}$ \\
\hline Test de Agilidad & 13 & 1.1 & 1.6 & 1.315 & 1345 \\
\hline $\begin{array}{l}\text { Test flexibilidad } \\
\text { extremidades superiores }\end{array}$ & 13 & 2.3 & 2.5 & 2.454 & .0660 \\
\hline $\begin{array}{l}\text { Test de equilibrio estático } \\
\text { pierna izquierda }\end{array}$ & 13 & 3.4 & 3.6 & 3.554 & .0660 \\
\hline $\begin{array}{l}\text { Test de equilibrio estático } \\
\text { pierna derecha }\end{array}$ & 13 & 4.5 & 4.6 & 4.577 & .0439 \\
\hline $\mathrm{N}$ válido (por lista) & 13 & & & & \\
\hline
\end{tabular}

En la tabla 1 se observa la evaluación estadística descriptiva en la cual el número de sujetos evaluados es de 13, para el test de agilidad la media fue de 1.315, test flexibilidad extremidades superiores con una media de 2.454, test de equilibrio estático pierna izquierda dio una media de 3.554 , test de equilibrio estático pierna derecha la media fue de 4.577

Edad.

Tabla 2. Rango de edad de los sujetos participantes

\begin{tabular}{|l|l|l|l|l|}
\hline & Frecuencia & Porcentaje & $\begin{array}{l}\text { Porcentaje } \\
\text { válido }\end{array}$ & $\begin{array}{l}\text { Porcentaje } \\
\text { acumulado }\end{array}$ \\
\hline $60-65$ & 4 & 30.8 & 30.8 & 30.8 \\
$66-70$ & 5 & 38.5 & 38.5 & 69.2 \\
$76-80$ & 4 & 30.8 & 30.8 & 100.0 \\
Total & 13 & 100.0 & 100.0 & \\
\hline
\end{tabular}

Se pude observar en la tabla 2 el predominio de edad de los sujetos participantes en su gran mayoría el rango de edad fue de 60 a 65 y 76 a 80 años, en este grupo observamos que están 8 sujetos, 
Núm. 28

\section{Académica sin Frontera}

ISSN: 2007-8870

\section{$\underline{\text { http://revistainvestigacionacademicasinfrontera.com }}$}

mientras en el los 66 a 70 años solo tenemos 5 sujetos. En su totalidad se incluyeron para participar en el trabajo 13 personas.

Sexo de los sujetos participantes en el programa.

Tabla 3. Sexo de los sujetos participantes.

\begin{tabular}{|l|l|l|l|l|}
\hline & & & Porcentaje & Porcentaje \\
& Frecuencia & Porcentaje & válido & acumulado \\
\hline Femenino & 12 & 92.3 & 92.3 & 92.3 \\
Masculino & 1 & 7.7 & 7.7 & 100.0 \\
Total & 13 & 100.0 & 100.0 & \\
\hline
\end{tabular}

En la tabla 3 y el grafico 3 se puede observar la prevalencia de sujetos con respectos al sexo. Siendo en mayor proporción el sexo femenino con 12 sujetos, mientras que para la realización del programa de acondicionamiento solo se contó con la participación de 1 sujeto.

Resultados del test de agilidad.

Tabla 4. Resultados del test de agilidad.

\begin{tabular}{|l|l|l|l|l|}
\hline & & & $\begin{array}{l}\text { Porcentaje } \\
\text { válido }\end{array}$ & $\begin{array}{l}\text { Porcentaje } \\
\text { acumulado }\end{array}$ \\
\cline { 2 - 5 } & Frecuencia & Porcentaje & 7.7 & 7.7 \\
\hline 5 seg. Excelente & 1 & 7.7 & 30.8 & 38.5 \\
6 seg. muy bueno & 4 & 30.8 & 15.4 & 53.8 \\
7 seg. Bueno & 2 & 15.4 & 38.5 & 92.3 \\
8 seg. Regular & 5 & 38.5 & 7.7 & 100.0 \\
10 seg. muy malo & 1 & 7.7 & 100.0 & \\
Total & 13 & 100.0 &
\end{tabular}




\section{http://revistainvestigacionacademicasinfrontera.com}

Con respecto al test de agilidad aplicado en las primeras sesiones a los sujetos participantes con el objetivo de conocer cómo se encontraban con respecto a esta habilidad se puede observar en la tabla 4 lo siguiente, solo 1 de los sujetos aparece con la peor evaluación para dicho test, mientras que en el apartado de regular se encuentra la gran mayoría de sujetos con un total de 5 personas. De igual manera podemos observar que la otra gran mayoría se encuentra en la calificación muy bueno con 4 sujetos con un tiempo de 6 segundos, hay que resaltar que solo 1 sujeto salió evaluado excelente con un tiempo de 5 segundos para el test de agilidad.

Resultados del test de extremidades superiores

Tabla 5. Resultados del test de flexibilidad extremidades superiores.

\begin{tabular}{|l|l|l|l|l|}
\hline & & & $\begin{array}{l}\text { Porcentaje } \\
\text { válido }\end{array}$ & $\begin{array}{l}\text { Porcentaje } \\
\text { acumulado }\end{array}$ \\
\hline 6-10 cm, bueno & 1 & 7.7 & 7.7 & 7.7 \\
$11-15 \mathrm{~cm}$, regular & 4 & 30.8 & 30.8 & 38.5 \\
$16-20 \mathrm{~cm}$, malo & 8 & 61.5 & 61.5 & 100.0 \\
Total & 13 & 100.0 & 100.0 & \\
\hline
\end{tabular}

El test de extremidades superiores que se aplicó en las primeras sesiones en la tabla 5 se puede observar que solo 1 sujeto salió en el rango de bueno, 4 sujetos resultaron en regular, y la mayoría de los sujetos salieron en la calificación de malo con una puntuación de 16-20cm. 


\section{$\underline{\text { http://revistainvestigacionacademicasinfrontera.com }}$}

Resultados del test de equilibrio estático pierna izquierda.

Tabla 6. Resultados del test de equilibrio estático pierna izquierda.

\begin{tabular}{|c|c|c|c|c|c|}
\hline & & Frecuencia & Porcentaje & $\begin{array}{l}\text { Porcentaje } \\
\text { válido }\end{array}$ & $\begin{array}{l}\text { Porcentaje } \\
\text { acumulado }\end{array}$ \\
\hline \multirow[t]{4}{*}{ Válide } & 20-29 seg. Regular & 1 & 7.7 & 7.7 & 7.7 \\
\hline & 10-19 seg. Malo & 4 & 30.8 & 30.8 & 38.5 \\
\hline & $0-9$ seg. muy malo & 8 & 61.5 & 61.5 & 100.0 \\
\hline & Total & 13 & 100.0 & 100.0 & \\
\hline
\end{tabular}

Con respecto al test de equilibrio estático se evaluó en ambas piernas para conocer con cuál de las piernas los sujetos logran tener un mejor equilibrio, primeramente obtuvimos los resultados de la pierna izquierda, obteniendo como resultados, 1 sujeto para la calificación de regular, siendo en este caso la mejor calificación, 4 sujetos resultaron en la calificación de malo y la mayoría de los sujetos que fueron 8 obtuvieron una calificación de muy malo, con el rango de 0-9 seg.

Resultados del test de equilibrio estático con pierna derecha

Tabla 7. Resultados del test de equilibrio estático pierna derecha.

\begin{tabular}{|l|l|l|l|l|}
\hline & Frecuencia & Porcentaje & $\begin{array}{l}\text { Porcentaje } \\
\text { válido }\end{array}$ & $\begin{array}{l}\text { Porcentaje } \\
\text { acumulado }\end{array}$ \\
\hline 10-19 seg. Malo & 3 & 23.1 & 23.1 & 23.1 \\
0-9 seg. muy malo & 10 & 76.9 & 76.9 & 100.0 \\
Total & 13 & 100.0 & 100.0 & \\
\hline
\end{tabular}

Para la evaluación del test de equilibrio de la pierna derecha en la tabla 7 las calificaciones resultaron 3 sujetos en calificación mala con un rango de 10-19 seg. Y en la otra calificación 10 sujetos resultaron en la calificación de muy malo con un rango de 0-9 seg. 


\section{http://revistainvestigacionacademicasinfrontera.com}

\section{Conclusiones y discusión}

Los adultos mayores descuidan con mayor frecuencia el realizar actividad física o ejercicio, es en gran parte por los cambios físicos y porque son muy pocos los profesionales de la salud que se enfocan en este tipo de población. Con los resultados mostrados en las tablas 4, 5, 6, 7 se concluye que los adultos mayores de INAPAM tienen una aptitud fisca deficiente, recomendamos implementar programas de ejercicios enfocados a mejorar la calidad de vida y aumentar la aptitud física de las personas de la tercera edad.

\section{Referencias}

Daza, K. D. (22 de Enero de 2011). Vejez y envejecimiento . Obtenido de grupo de investigacion en actividad fisica y desarrollo humano : http://www.urosario.edu.co/urosario_files/dd/dd857fc5-5a01-4355-b07ae2f0720b216b.pdf

IAAM. (30 de marzo de 2018). http://www.adultomayor.cdmx.gob.mx. Recuperado el 14 de noviembre de 2017, de http://www.adultomayor.cdmx.gob.mx/index.php/quienes-el-adulto-mayor

Havighurst, R. J. (1948). Developmental tasks and education. New York: McKay.

Jupiter Ramos, E., Meza Calleja, A. M., Maldonado Hernandez, I., Ortega Medellin, M. P., \& Hernandez Paz, T. M. (11 de diciembre de 2009). Aportes de una conceptualizacion de la vejez. Obtenido de aportes de una conceptualizacion de la vejez: 


\section{http://revistainvestigacionacademicasinfrontera.com}

http://www.cucs.udg.mx/revistas/edu_desarrollo/anteriores/11/011_Ramos.pdf

OMS. (2015). Informe mundial sobre el envejecimiento y la salud. Ginebra, Suiza: ePub. Obtenido de http://apps.who.int/iris/bitstream/handle/10665/186466/9789240694873_spa.pdf;j sessionid=7E09DD908F2E61EA3D3752528536701A ? sequence $=1$

OPS. (30 de Septiembre de 2015). www.paho.org. Obtenido de https://www.paho.org/hq/index.php?option=com_content\&view=article\&id=11302 $\% 3$ Aworld-population-over-60-to-double-2050\&catid=740\%3Apressreleases\&ltemid $=1926 \&$ lang $=\mathrm{es}$

Ceballos, G. O., Alvarez, B. J., \& Medina, R. R. (2012). Actividad fisica y calidad de vida en adultos mayores. Obtenido de http://eprints.uanl.mx/4476/1/Capitulos\%20de\%20libro.pdf

Quevedo, T. E. (mayo-agosto de 2011). Caidas en adultos mayores. (U. J. Tabasco, Ed.) Horizonte Sanitario, 10(2), 4-6.

Guzman, A. Q. (Noviembre de 2010). Universidad Autonoma del Estado de Hidalgo. Obtenido de Analisis de la calidad de vida en adultos mayores del municipio de Tetepango Hidalgo:

https://www.uaeh.edu.mx/nuestro_alumnado/esc_sup/actopan/licenciatura/Analis is\%20de\%20la\%20calidad\%20de\%20vida.pdf

Moreno, G. A. (2005). Incidencia de la Actividad Física en el adulto mayor. Revista Internacional de Medicina y Ciencias de la Actividad Física y el Deporte, 5(19), 222-237.

Fernandez, G. A. (2010). Deporte y ejercicio fisico en las personas mayores. Recuperado el 21 de Agosto de 2018, de 


\section{http://revistainvestigacionacademicasinfrontera.com}

http://www.revista60ymas.es/InterPresent1/groups/revistas/documents/binario/s2 94afondo.pdf

OMS. (2018). Estrategia mundial sobre régimen alimentario, actividad física y salud. Obtenido de La actividad física en los adultos mayores: http://www.who.int/dietphysicalactivity/factsheet_olderadults/es/

OMS. (2018). La actividad fisica en los adultos mayores. Obtenido de Niveles recomendados de actividad fisica para la salud de 65 años en adelante: http://www.who.int/dietphysicalactivity/factsheet_olderadults/es/

OMS. (23 de Febrero de 2018). Actividad física; Datos y cifras. Obtenido de http://www.who.int/es/news-room/fact-sheets/detail/physical-activity

Perez, L. A., \& Perez, F. A. (Diciembre de 2013). El sedentarismo. Un problema educativo y social. efdeportes.com, 187. Recuperado el 21 de Junio de 2018, de http://www.efdeportes.com/efd187/el-sedentarismo-un-problema-educativo-ysocial.htm

Cossio, B. M., \& Arruda, M. (2009). Propuesta de valores normativos para la evaluacion de la aptitud fisica en niños de 6 a 12 años de Arequipa, Peru. revista medica herediana, 4(20). Recuperado el 21 de Junio de 2018, de http://www.upch.edu.pe/vrinve/dugic/revistas/index.php/RMH/article/view/1005/0

Laverde, R. G., Esguerra, G. A., Espinosa, J. C., \& Lozano, G. D. (15 de Enero-Junio de 2011). Aptitud fisica y salud de corredores aficionados. Hallazgos, 8(15), $215-$ 235. Recuperado el 21 de Junio de 2018, de http://www.redalyc.org/pdf/4138/413835204012.pdf

Guillen, P. L., Bueno, F. E., Gutierrez, C. M., \& Guerra, S. E. (2012). Programa de actividad física y su incidencia en la depresión y bienestar subjetivo de adultos 
(Julio- Diciembre 2018)

Núm. 28

ISSN: 2007-8870

http://revistainvestigacionacademicasinfrontera.com

mayores. Retos. Recuperado el 21 de Junio de 2018, de

https://recyt.fecyt.es/index.php/retos/article/view/49638

UE. (10 de Octubre de 2008). ec.europa.eu. Obtenido de

http://ec.europa.eu/assets/eac/sport/library/policy documents/eu-physicalactivity-guidelines-2008 es.pdf

\section{Como citar este articulo}

José Samuel Borbón Vega- Jesús Santos Cantúa Quintero- Emmanuel Josef García Medina- Marco Alejandro Molina Rodríguez. Evaluación de la aptitud física en adultos mayores miembros del programa INAPAM de la ciudad de Navojoa Sonora. RIASF. Núm. 28, Julio-diciembre (2018), ISSN 2007-8870. pp. xx-xx. 